\title{
Safety and efficacy of brainstem biopsy in children and young adults
}

\author{
David S. Hersh, MD, ${ }^{1,2}$ Rahul Kumar, PhD, MS, ${ }^{3}$ Kenneth A. Moore, MD, ${ }^{3}$ Luke G. F. Smith, MD, ${ }^{4}$ \\ Christopher L. Tinkle, MD, PhD, ${ }^{5}$ Jason Chiang, MD, PhD, ${ }^{6}$ Zoltan Patay, MD, PhD, ${ }^{7}$ \\ Amar Gajjar, MD, ${ }^{8}$ Asim F. Choudhri, MD, ${ }^{3,9-11}$ Jorge A. Lee-Diaz, MD, ${ }^{3,9-11}$ Brandy Vaughn, RN, ${ }^{11}$ \\ and Paul Klimo Jr., MD, MPH3,11,12
}

\begin{abstract}
'Division of Neurosurgery, Connecticut Children's, Hartford; 'Department of Surgery, UConn School of Medicine, Farmington, Connecticut; ${ }^{3}$ Department of Neurosurgery, University of Tennessee Health Science Center, Memphis, Tennessee; ${ }^{4}$ Department of Neurosurgery, The Ohio State University, Columbus, Ohio; Departments of ${ }^{5}$ Radiation Oncology, ${ }^{6}$ Pathology, and ${ }^{7}$ Diagnostic

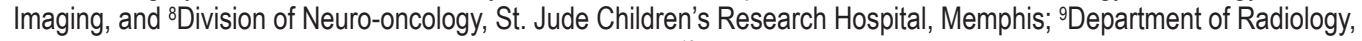
University of Tennessee Health Science Center, Memphis; ${ }^{10}$ Division of Neuroradiology, Le Bonheur Neuroscience Institute, Memphis; ${ }^{11}$ Le Bonheur Children's Hospital, Memphis; and ${ }^{12}$ Semmes Murphey, Memphis, Tennessee
\end{abstract}

OBJECTIVE Biopsies of brainstem lesions are performed to establish a diagnosis in the setting of an atypical clinical or radiological presentation, or to facilitate molecular studies. A better understanding of the safety and diagnostic yield of brainstem biopsies would help guide appropriate patient selection.

METHODS All patients who underwent biopsy of a brainstem lesion during the period from January 2011 to June 2019 were reviewed. Demographic, radiological, surgical, and outcome data were collected.

RESULTS A total of 58 patients underwent 65 brainstem biopsies during the study period. Overall, the median age was 7.6 years (IQR 3.9-14.2 years). Twenty-two of the 65 biopsies (34\%) were open, $42(65 \%)$ were stereotactic, and 1 was endoscopic. In 3 cases (5\%), a ventriculoperitoneal shunt was placed, and in 9 cases (14\%), a posterior fossa decompression was performed during the same operative session as the biopsy. An intraoperative MRI (iMRI) was performed in 28 cases (43\%). In 3 of these cases (11\%), the biopsy was off target and additional samples were obtained during the same procedure. New neurological deficits were noted in 5 cases (8\%), including sensory deficits, ophthalmoparesis/ nystagmus, facial weakness, and hearing loss; these deficits persisted in 2 cases and were transient in 3 cases. A pseudomeningocele occurred in 1 patient; no patients developed a CSF leak or infection. In 8 cases (13\%) an additional procedure was needed to obtain a diagnosis.

CONCLUSIONS Brainstem biopsies are safe and effective. Target selection and approach should be a collaborative effort. iMRI can be used to assess biopsy accuracy in real time, thereby allowing any adjustment if necessary.

https://thejns.org/doi/abs/10.3171/2020.4.PEDS2092

KEYWORDS brainstem; biopsy; stereotactic; intraoperative MRI; oncology

$\mathrm{B}$ RAINSTEM tumors are relatively rare in adults but are found at a much higher rate in children, comprising approximately $10 \%-20 \%$ of pediatric brain tumors. ${ }^{1-3}$ Diffuse intrinsic pontine gliomas (DIPGs) are the most common subtype, accounting for up to $80 \%$ of pediatric brainstem gliomas, and have a dismal prognosis with a mean survival of less than 12 months and a 2 -year survival rate of less than $10 \%$., $^{1,-6}$ The remaining brainstem tumors include low-grade gliomas (most notably pilocytic astrocytomas), non-DIPG midline high-grade gliomas, embryonal tumors such as embryonal tumor with multilayered rosettes (ETMR), and other nonneoplastic entities. ${ }^{7-15}$

Stereotactic biopsies of brainstem lesions were first described in 1978 in adults and were performed frequently during the 1980s in both adult and pediatric patients, with a number of reports documenting a relatively high diagnostic yield, as well as a low complication rate. ${ }^{16-21}$ As the

ABBREVIATIONS ADC = apparent diffusion coefficient; $A T R T=$ atypical teratoid rhabdoid tumor; DIPG = diffuse intrinsic pontine glioma; $D S C=$ dynamic susceptibility contrast; DWI = diffusion-weighted imaging; ETMR = embryonal tumor with multilayered rosettes; iMRI = intraoperative MRI; MRS = magnetic resonance spectroscopy; NAA $=\mathrm{N}$-acetylaspartate

SUBMITTED February 19, 2020. ACCEPTED April 29, 2020.

INCLUDE WHEN CITING Published online July 31, 2020; DOI: 10.3171/2020.4.PEDS2092. 
resolution of MRI improved, however, the role of biopsy became controversial, particularly for patients with diffusely infiltrative lesions for whom treatment options have historically been limited to radiotherapy. As a result, in 1993 Albright et al. summarized a report from the Children's Cancer Group and concluded that given the characteristic appearance of DIPGs on MRI, MR scans should replace biopsies for DIPG diagnosis, and DIPG patients should proceed to radiation therapy without a histological diagnosis. ${ }^{22}$

Over 25 years later, this topic remains highly controversial. Some studies have identified significant interobserver variation in interpreting imaging studies of brainstem lesions, and others have identified a high sensitivity but low specificity in differentiating tumors from other diseases of the brainstem based on clinical findings and MRI characteristics. ${ }^{23-25}$ Additionally, the ever-changing field of molecular neuro-oncology has allowed the identification of mutations that are potentially amenable to targeted therapies but can only be determined through individual molecular profiles obtained from biopsy tissue. ${ }^{26-29}$ As a result, the debate over whether to biopsy diffusely infiltrative brainstem lesions has been renewed in recent years. Regardless, there remains consensus that lesions with atypical clinical presentations or radiological features, including a focal appearance and/or avid or extensive contrast enhancement, should be biopsied. . $^{12,14,15,22,25,29}$

As a result of this ongoing controversy, it remains necessary to define the safety and efficacy of biopsy of brainstem lesions. A number of advances have been incorporated since those early studies, including frameless stereotactic navigation, advanced imaging techniques for selecting a target, and the use of intraoperative MRI (iMRI) at some centers to confirm the biopsy site in real time. Here, we describe our multidisciplinary approach to pediatric patients and young adults with brainstem lesions-how we decide when a biopsy is indicated, whether to perform an open or stereotactic procedure, and how to select a target.

\section{Methods \\ Study Population}

Our institutional review board (IRB) approved this study (IRB no. 18-06319-XP STJUDE); the requirement for informed consent from the patient or guardian was waived by the IRB. All patients who underwent biopsy of a lesion involving the midbrain, pons, medulla, or cerebellar peduncle between January 2011 and June 2019 were included. Each biopsy procedure, whether open or stereotactic, was studied individually. Demographic, clinical, radiological, surgical, and follow-up data were collected.

\section{Patient Evaluation and Management}

Patients diagnosed with a brainstem lesion were discussed in a weekly multidisciplinary conference involving neuro-oncologists, radiation oncologists, neurosurgeons, neuroradiologists, and neuropathologists (Fig. 1). A biopsy was recommended for any lesion with an "atypical" radiographic appearance and/or an unusual clinical course. Alternatively, if tissue was required for enrollment in a clinical trial, then a biopsy was recommended.
Possible biopsy sites were evaluated preoperatively by group discussion, primarily between the neurosurgeon and neuroradiologist(s), with additional input from the neuro-oncologist or radiation oncologist. When considering possible sites, the emphasis was on maximizing both the safety and diagnostic yield of the biopsy. Areas of contrast enhancement and/or hypercellularity (as indicated by relative restricted diffusion identified on diffusion-weighted imaging [DWI] sequences and the apparent diffusion coefficient [ADC] map) were often selected as biopsy targets. In cases in which advanced imaging was performed, regions with the following findings were prioritized: altered metabolic activity (as indicated by a high choline $/ N$-acetylaspartate $[\mathrm{NAA}]$ ratio on magnetic resonance spectroscopy [MRS]) and/or increased blood flow (as demonstrated on dynamic susceptibility contrast [DSC] perfusion MRI; Fig. 2). Target location and the surgical approach/trajectory were carefully assessed in relation to eloquent and vital anatomical structures, often with the assistance of tractography.

A stereotactic needle biopsy was performed in cases for which the target was deemed to be reasonably sized and safely accessible; otherwise, an open biopsy was pursued. In some cases, an iMRI scan was performed; the decision to utilize the iMRI depended on the operating surgeon and availability of the scanner. If the iMRI demonstrated that the biopsy sample was from outside of the lesion (both off-lesion and off-target) or intended target (intralesion but off-target), or the frozen pathology results were inconclusive, the process above was repeated and either another stereotactic biopsy was attempted or conversion to an open approach was performed.

\section{Statistical Analysis}

Descriptive statistics were reported, including median and interquartile range (IQR) for continuous data, and frequency and percentage for categorical data.

\section{Results \\ Patient Population}

A total of 65 procedures in 58 patients met the study criteria. Demographic and clinical features are summarized in Table 1. Of the 65 procedures, 39 were in male patients $(60 \%)$ and 26 were in female patients $(40 \%)$. The median age at the time of biopsy was 7.5 years (IQR 3.914.1 years). Thirteen of 58 patients $(22.4 \%)$ had a prior oncological history, and the indication for the current biopsy was to differentiate recurrence or progression from treatment-related effects. Prior tumor diagnoses included DIPG in 2 patients (3.4\%), ependymoma in 2 (3.4\%), medulloblastoma in $2(3.4 \%)$, pilocytic astrocytoma in 2 (3.4\%), tectal plate glioma in $2(3.4 \%)$, high-grade glioma in $1(1.7 \%)$, atypical teratoid rhabdoid tumor (ATRT) in $1(1.7 \%)$, and pilomyxoid astrocytoma in 1 patient (1.7\%). Eleven patients $(19.0 \%)$ had undergone prior local radiation, $6(10.3 \%)$ had been treated previously with chemotherapy, and $10(17.2 \%)$ had undergone prior resection.

\section{Radiological Features}

Radiological features are summarized in Table 2. All 
DIAGNOSIS

Brainstem

mass

INDICATION

FOR BIOPSY

\section{Multi-disciplinary tumor board}

Tissue requirement for trial? Atypical radiographic appearance? Unusual clinical features/course?
No Non-surgical management

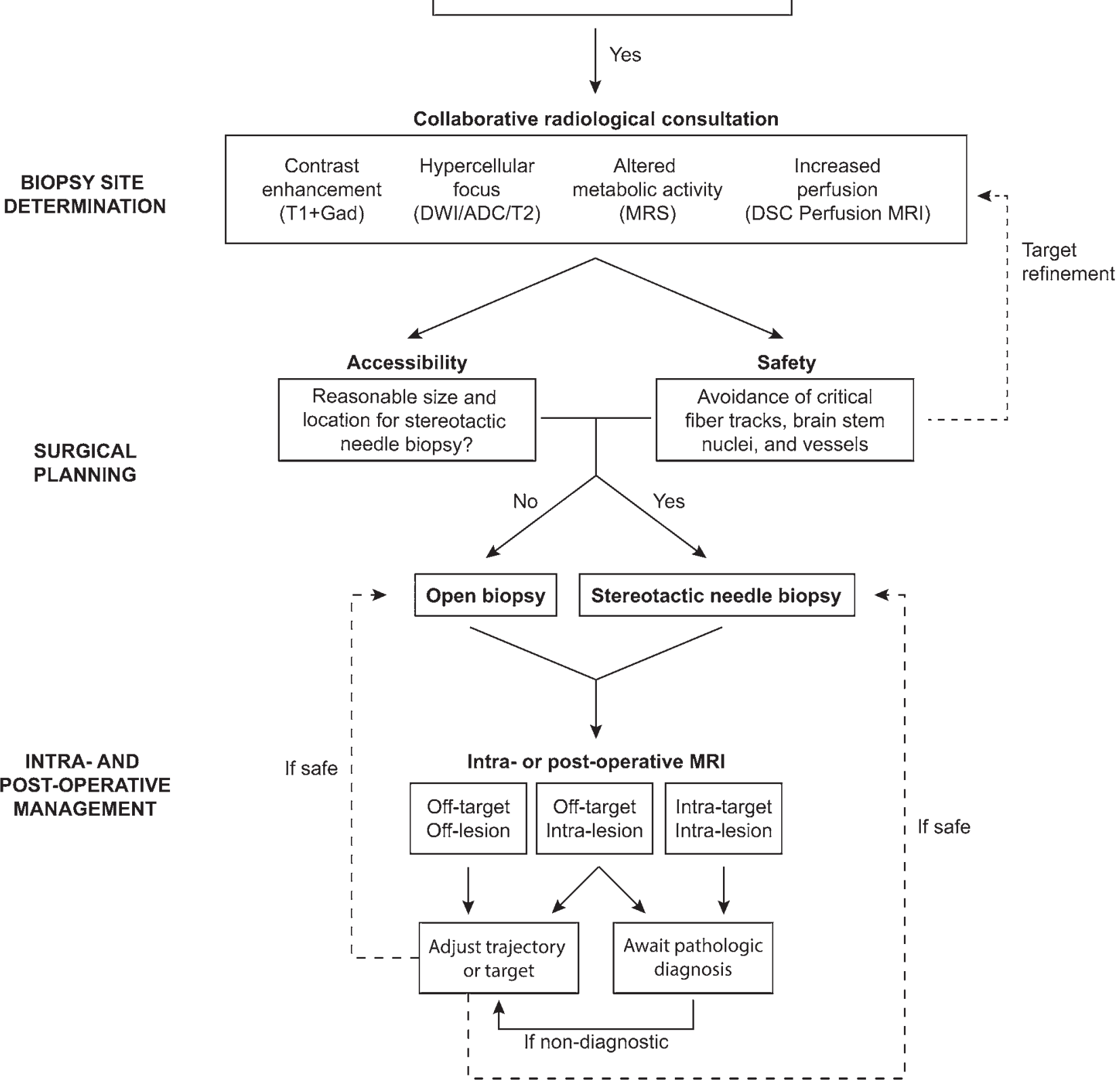

FIG. 1. Workflow diagram for the multidisciplinary evaluation of pediatric patients with brainstem lesions. The first step involves determining which patients should undergo a biopsy; subsequent decisions include choosing an appropriate biopsy site and determining whether to perform an open biopsy or a stereotactic needle biopsy. Gad = gadolinium. 

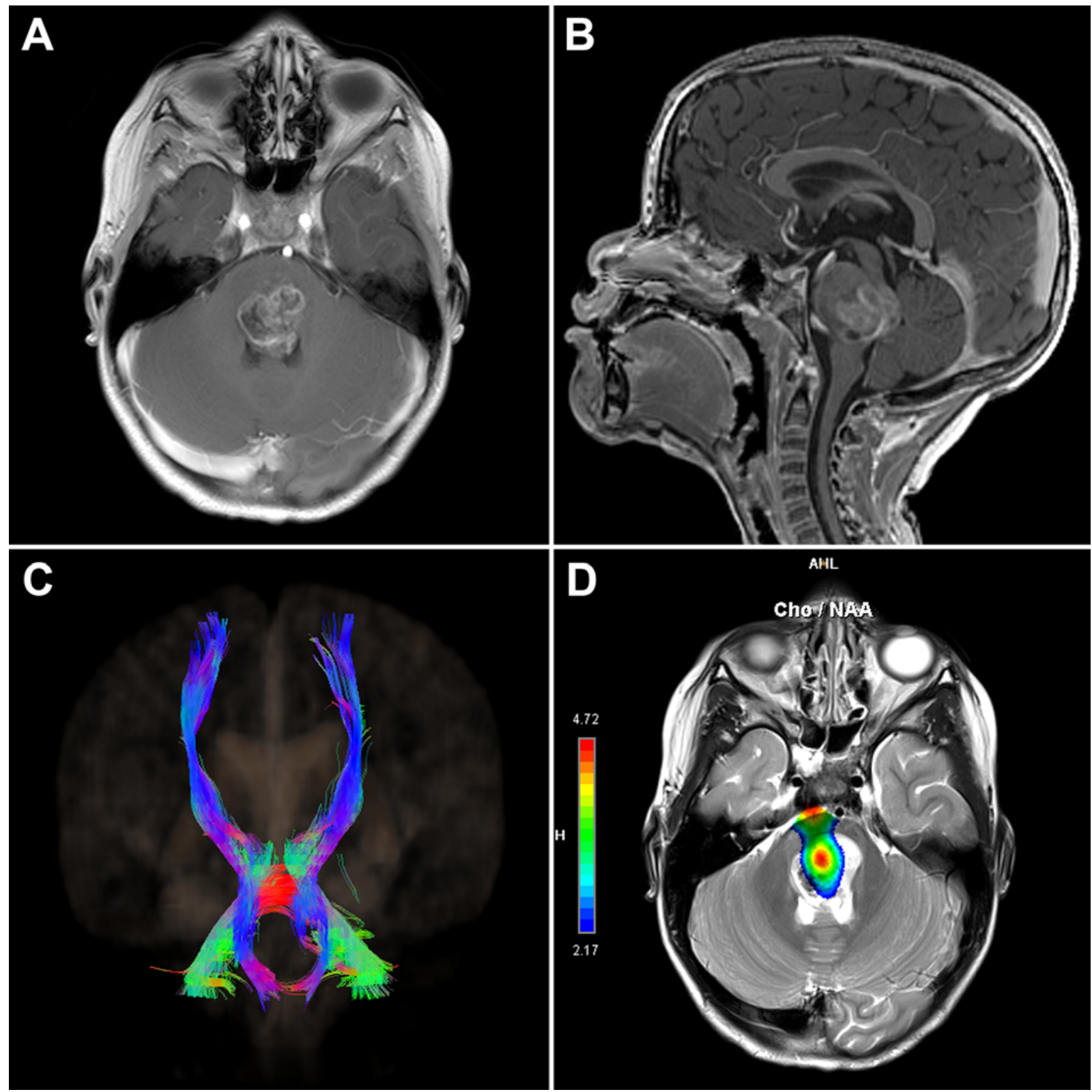

FIG. 2. A representative example of the target selection process. The patient was a 4-year-old boy who presented with a pontine mass that was atypical for a DIPG. He underwent a stereotactic needle biopsy that demonstrated abundant mitotic activity, necrosis, and the histone H3K27M mutation, resulting in a diagnosis of diffuse midline glioma. Axial (A) and sagittal (B) T1-weighted contrast-enhanced sequences demonstrated an approximately $3-\mathrm{cm}$ relatively discrete mass in the dorsal aspect of the pons, with heterogeneous enhancement and areas of necrosis. (C) Tractography demonstrated splaying of the surrounding fibers of the corticospinal and central segmental tracts. (D) MRS was used to generate a metabolic map that demonstrated the region with the highest choline/NAA ratio, which was selected as the biopsy target. Figure is available in color online only.

lesions involved the brainstem with or without involvement of adjacent regions. The lesion directly involved or extended to the midbrain in 22 of the 65 biopsies (33.8\%), the pons in $51(78.5 \%)$, the medulla in $26(40 \%)$, the cerebellar peduncle in $34(52.3 \%)$, the cerebellar hemisphere in $11(16.9 \%)$, the thalamus in $4(6.2 \%)$, and the cervical spine in $7(10.8 \%)$. Twenty-one lesions $(32.3 \%)$ were characterized as discrete, $42(64.6 \%)$ were characterized as infiltrating, and 2 (3.1\%) had features of both. Forty-two lesions $(64.6 \%)$ demonstrated contrast enhancement and 23
(35.4\%) were nonenhancing. Twenty-eight lesions (43.1\%) demonstrated measurable diffusion restriction, 36 (55.4\%) demonstrated facilitated diffusion, and $1(1.5 \%)$ did not have DWI sequences preoperatively. Twenty-eight lesions (43.1\%) had an exophytic portion, which was typically the site that was targeted for biopsy. Six lesions (9.2\%) had imaging that was characteristic of a DIPG.

In 4 cases $(6.2 \%)$, advanced preoperative imaging was specifically utilized to select the biopsy site. In 2 of these cases, MRS was used to identify a focus with a high cho- 
TABLE 1. Demographic and clinical features

\begin{tabular}{lc}
\hline \multicolumn{1}{c}{ Variable } & Value \\
\hline Male* $^{*}$ & $39(60.0)$ \\
\hline Median age at biopsy, yrs (IQR) & $7.5(3.9-14.1)$ \\
\hline Prior oncologic history $\dagger$ & $13(22.4)$ \\
\hline Prior diagnosis $†$ & $2(3.4)$ \\
\hline DIPG & $2(3.4)$ \\
\hline Ependymoma & $2(3.4)$ \\
\hline Medulloblastoma & $2(3.4)$ \\
\hline Pilocytic astrocytoma & $2(3.4)$ \\
\hline Tectal plate glioma & $1(1.7)$ \\
\hline High-grade glioma & $1(1.7)$ \\
\hline ATRT & $1(1.7)$ \\
\hline Pilomyxoid astrocytoma & $11(19.0)$ \\
\hline Prior treatment† & $6(10.3)$ \\
\hline Radiation & $10(17.2)$ \\
\hline Chemotherapy
\end{tabular}

*Values based on number (\%) of the total procedures $(n=65)$.

$\dagger$ Values based on number $(\%)$ of the total included patients $(n=58)$.

line/NAA ratio, while in the other 2 cases DSC perfusion MRI was used to identify an area of increased perfusion. There were other cases in which advanced imaging was obtained; however, in these cases the images were not specifically used to select a biopsy target because the location was considered too risky, there was an exophytic portion of the tumor that was safer to biopsy, or other radiological features, such as contrast enhancement or diffusion restriction, were used to select the biopsy target instead. In those cases in which advanced imaging was critical to determining the biopsy target, the data obtained from DWI, MRS, and DSC were concordant.

\section{Surgical Features}

Surgical features are summarized in Table 3. Twentytwo of the procedures (33.8\%) were open biopsies, 42 $(64.6 \%)$ were stereotactic biopsies, and $1(1.5 \%)$ was an endoscopic intraventricular biopsy. Of the open biopsies, $17(77.3 \%)$ were performed via a midline suboccipital approach, 2 (9.1\%) via an extended suboccipital approach, $1(4.5 \%)$ via a middle temporal gyrus approach, $1(4.5 \%)$ via a pterional transsylvian approach, and 1 (4.5\%) via an occipital interhemispheric approach. All stereotactic biopsies were performed via an infratentorial approach. Depending on surgeon preference, the selected target, and the patient's body habitus, biopsy specimens were obtained with the patients in the following positions: 21 $(50 \%)$ in the prone position, $17(40.5 \%)$ in the supine position with the head turned, and $4(9.5 \%)$ in the lateral position. Almost all of the procedures (60 procedures, or $92 \%$ of the total) were performed by 1 of 2 surgeons. In 16 cases $(24.6 \%)$, another procedure was performed concomitantly in the same setting as the biopsy. These concomitant procedures included shunt placement in 3 cases (4.6\%), endoscopic third ventriculostomy in 1 case (1.5\%),
TABLE 2. Radiological features ( $n=65$ procedures)

\begin{tabular}{cc}
\hline \multicolumn{1}{c}{ Variable } & No. of Procedures $(\%)$ \\
\hline Region* & \\
\hline Midbrain & $22(33.8)$ \\
\hline Pons & $51(78.5)$ \\
\hline Medulla & $26(40.0)$ \\
\hline Cerebellar peduncle & $34(52.3)$ \\
\hline Cerebellar hemisphere & $11(16.9)$ \\
\hline Thalamus & $4(6.2)$ \\
\hline Cervical spine & $7(10.8)$ \\
\hline Focality & $21(32.3)$ \\
\hline Discrete & $42(64.6)$ \\
\hline Infiltrative & $2(3.1)$ \\
\hline Features of both & $42(64.6)$ \\
\hline Enhancement & $23(35.4)$ \\
\hline Enhancing & \\
\hline Nonenhancing & $28(43.1)$ \\
\hline Diffusion & $36(55.4)$ \\
\hline Restricted & $1(1.5)$ \\
\hline Facilitated & $28(43.1)$ \\
\hline Unknown & $6(9.2)$ \\
\hline Exophytic & \\
\hline Typical for DIPG & \\
\hline
\end{tabular}

* Each lesion may have spanned one or more anatomic regions.

posterior fossa decompression in 9 cases (13.8\%), and a variety of miscellaneous procedures in 4 cases $(6.2 \%)$, including simultaneous open biopsy of a second cerebellar lesion, repair of a pseudomeningocele, mesh cranioplasty, and fenestration of a pontine cyst.

Among the 42 stereotactic biopsies, a single trajectory was used in 36 cases $(85.7 \%$ ), while 2 trajectories were utilized in 5 cases (11.9\%), and 3 trajectories were used in 1 case $(2.4 \%)$. There were an additional 6 cases $(14.3 \%)$ in which biopsies were obtained at 2 depths along a single trajectory. The total number of core samples that were obtained, when recorded, ranged from 2 to 14. Aspirate containing blood products was specifically mentioned in the operative report in 3 cases $(7.1 \%)$.

Among the 65 procedures, an iMRI was performed in 28 cases (43.1\%). There were 2 cases (3.1\%) in which 2 iMRIs were performed. Among the stereotactic procedures, the biopsy needle was adjusted (either the trajectory or the depth) in 3 cases ( $4.6 \%$ of the overall cases, $10.7 \%$ of the iMRI cases). The median operative time was 3.0 hours (IQR 2.4-3.7 hours) for open biopsies and 1.6 hours (IQR 1.3-2.2 hours) for stereotactic biopsies, including iMRI time. The median inpatient length of stay was 5 days (IQR 3.3-6.8 days) for open biopsies and 2 days (IQR 1.3-3 days) for stereotactic biopsies.

\section{Safety}

Intraoperative and/or postoperative imaging was obtained for 52 of the 65 biopsies (80\%). Among those patients who underwent imaging, there were no clinically 
TABLE 3. Surgical features $(n=65$ procedures)

\begin{tabular}{|c|c|}
\hline Variable & Value \\
\hline \multicolumn{2}{|l|}{ Type of biopsy } \\
\hline Open & $22(33.8)$ \\
\hline Stereotactic & $42(64.6)$ \\
\hline Endoscopic & $1(1.5)$ \\
\hline \multicolumn{2}{|l|}{ Positioning } \\
\hline Prone & $40(61.5)$ \\
\hline Supine & $20(30.8)$ \\
\hline Lateral & $5(7.7)$ \\
\hline Concomitant procedures & $16(24.6)$ \\
\hline Shunt & $3(4.6)$ \\
\hline ETV & $1(1.5)$ \\
\hline Posterior fossa decompression & $9(13.8)$ \\
\hline Miscellaneous & $4(6.2)$ \\
\hline \multicolumn{2}{|l|}{ No. of trajectories* } \\
\hline 1 & $36(85.7) \dagger$ \\
\hline 2 & $5(11.9)$ \\
\hline 3 & $1(2.4)$ \\
\hline \multicolumn{2}{|l|}{ No. of iMRIs } \\
\hline 1 & $26(40.0)$ \\
\hline 2 & $2(3.1)$ \\
\hline Biopsy needle adjusted after iMRI ${ }^{*}$ & $3(4.6)$ \\
\hline \multicolumn{2}{|l|}{ Median operative time in hrs (IQR) } \\
\hline Open & $3.0(2.4-3.7)$ \\
\hline Stereotactic & $1.6(1.3-2.2)$ \\
\hline \multicolumn{2}{|l|}{ Median length of stay in days (IQR) } \\
\hline Open & $5(3.3-6.8)$ \\
\hline Stereotactic & $2(1.3-3.0)$ \\
\hline
\end{tabular}

ETV = endoscopic third ventriculostomy.

Values are presented as number of procedures (\%) unless otherwise indicated.

${ }^{*}$ For stereotactic needle biopsies only.

† There were an additional 6 cases (14.3\%) in which biopsies were obtained at

2 depths along a single trajectory.

significant hemorrhages noted on either intraoperative or postoperative imaging. New or worsening neurological issues were noted in 5 cases (7.7\%) as follows: 1) A 12-yearold girl who underwent open biopsy of a cervicomedullary ganglioglioma developed unilateral decreased sensation in the foot, though this resolved by the 4-month followup. 2) A 10-year-old boy who underwent open biopsy of a midbrain germinoma adjacent to the pineal gland via an occipital interhemispheric approach developed an upgaze palsy and dysconjugate gaze, although these issues were improving by discharge. 3) A 4-year-old girl who underwent a stereotactic needle biopsy of a pontine pilocytic astrocytoma developed new-onset cranial nerve VI and VII palsies on the right side, ultimately requiring a tarsorrhaphy and facial reanimation surgery. Her biopsy results demonstrated a KIAA1549-BRAF fusion and she was subsequently treated with a MEK inhibitor. 4) A 5-yearold girl who underwent a stereotactic needle biopsy of a pontomedullary pilocytic astrocytoma developed transient left-sided hearing loss, nystagmus, and ataxia, although these symptoms resolved after 1 day. 5) A 3-year-old boy who underwent a stereotactic needle biopsy of a grade IV diffuse midline glioma involving the pons and midbrain developed worsening of his preoperative right-sided facial palsy (Fig. 3). He was found to have an H3K27M mutation, which made him eligible for a clinical trial involving a small-molecule inhibitor of class I PI3K and mTOR kinase. At last follow-up, all patients were alive with the exception of the last patient, who died of disease progression 10 months after the biopsy. Additionally, among the 65 biopsies there was 1 pseudomeningocele $(1.5 \%), 1$ case (1.5\%) of wound breakdown, and no CSF leaks or infections. One patient $(1.5 \%)$ had a delayed subdural hematoma secondary to overshunting.

\section{Efficacy and Long-Term Outcomes}

Of the 65 biopsies, $53(81.5 \%)$ yielded samples that were diagnostic for tumor. This included 20 of the 22 open biopsies (90.9\%), 32 of the 42 stereotactic biopsies (76.2\%), and the 1 endoscopic biopsy. In another 4 cases (1 open and 3 stereotactic cases), a nononcologic diagnosis was confirmed in each case, and diagnoses included 1 case of deep white matter edema, 1 infarct, and 2 cases of treatment-related changes. One patient was diagnosed with a hamartoma during a subsequent resection. The final diagnoses are summarized in Table 4. Overall, one or more additional procedures were required in order to obtain diagnostic tissue following 1 open and 7 stereotactic cases, for a total of 8 cases (12.3\%). Of these, 7 patients required a second procedure and 1 patient required a third procedure (Fig. 4). Diagnoses obtained via a subsequent resection were considered diagnostic failures of the initial biopsy. The overall diagnostic yield was therefore $95.5 \%$ (21 of 22) for open biopsies and 83.3\% (35 of 42) for stereotactic biopsies.

Overall, the median duration of follow-up was 28.3 months (IQR 6.9-71.1 months). Chemotherapy was administered following 31 biopsies (47.7\%), radiation therapy was performed following 35 biopsies (53.8\%), and surgical resection was pursued following 8 biopsies (12.3\%). Of the 58 patients, $18(31 \%)$ were treated with molecularly targeted therapies.

\section{Discussion}

The utility of performing a biopsy of an intrinsic brainstem tumor has been actively debated over the past 3 decades. Those who have argued against performing a biopsy have emphasized that given the degree of heterogeneity present within many infiltrative tumors, the small sample obtained from a stereotactic biopsy is not necessarily representative of the entire tumor, resulting in a low diagnostic yield..$^{8,25,30,31}$ Particularly for tumors that are clinically and radiologically classic for a DIPG, imaging advances have seemed to increase the sensitivity and specificity of MRI to the point that the diagnosis can be made with imaging alone..$^{22,32}$ In a study of 142 pediatric patients with brainstem disease, radiologists were able to correctly classify lesions as tumors or other diseases of the brainstem in $95 \%-99 \%$ of cases based on MRI alone. ${ }^{23}$ When considered in the context of the poor prognosis of 

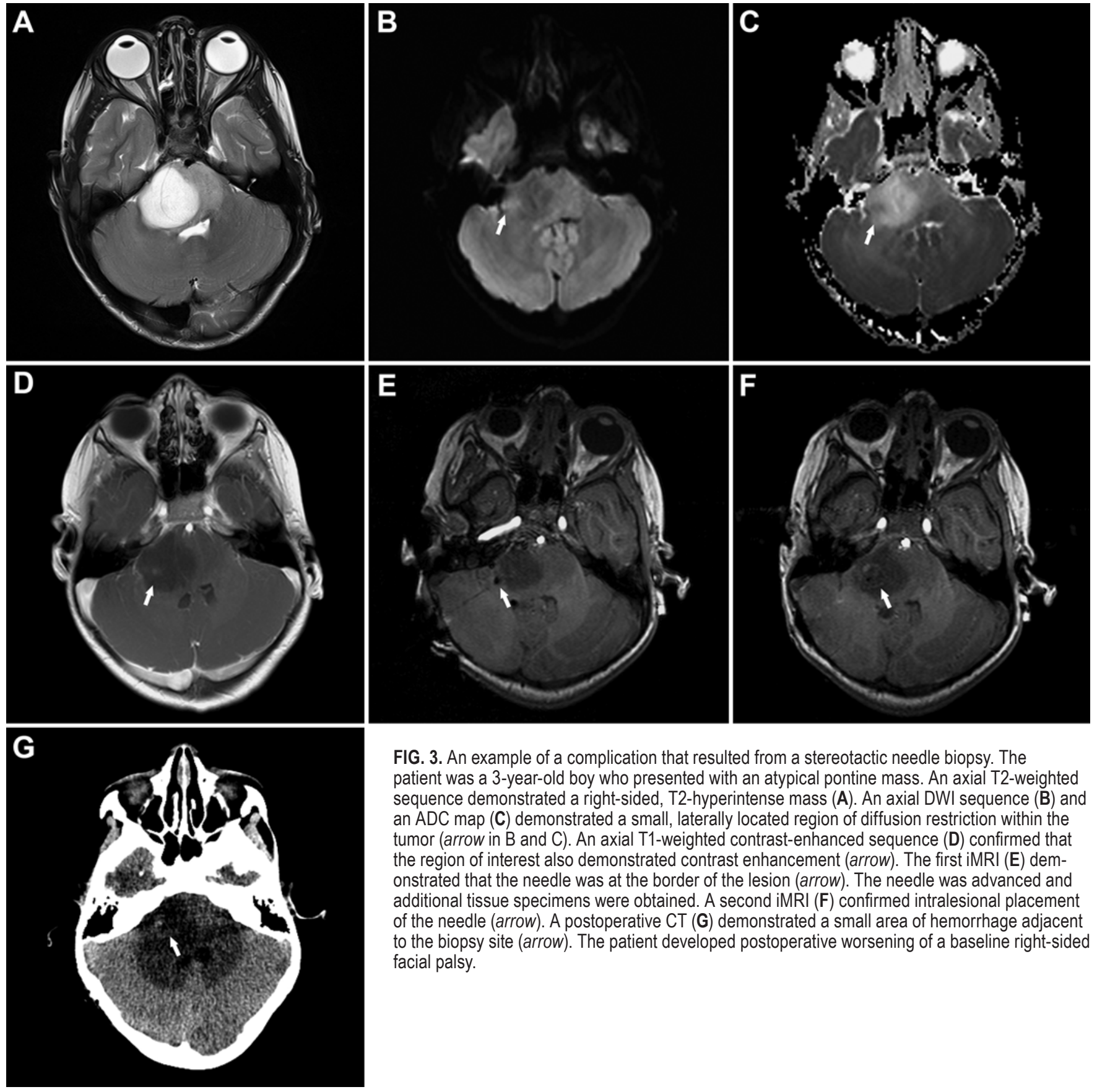

FIG. 3. An example of a complication that resulted from a stereotactic needle biopsy. The patient was a 3-year-old boy who presented with an atypical pontine mass. An axial T2-weighted sequence demonstrated a right-sided, T2-hyperintense mass (A). An axial DWI sequence (B) and an ADC map (C) demonstrated a small, laterally located region of diffusion restriction within the tumor (arrow in B and C). An axial T1-weighted contrast-enhanced sequence (D) confirmed that the region of interest also demonstrated contrast enhancement (arrow). The first iMRI (E) demonstrated that the needle was at the border of the lesion (arrow). The needle was advanced and additional tissue specimens were obtained. A second iMRI (F) confirmed intralesional placement of the needle (arrow). A postoperative CT (G) demonstrated a small area of hemorrhage adjacent to the biopsy site (arrow). The patient developed postoperative worsening of a baseline right-sided facial palsy.

these patients, and the fact that radiation therapy remains the standard treatment option for these patients regardless of the grade of the tumor, the risks of a biopsy are felt by some clinicians to be unwarranted.

Conversely, some groups have advocated for an increased role for surgical biopsy in the workup and management of brainstem lesions. In contrast to the high sensitivity of MRI cited in the reported study above, ${ }^{23}$ Rachinger et al. ${ }^{33}$ demonstrated that in adults, MRI had a specificity of $46.6 \%$ and sensitivity of $62.5 \%$ for diagnosing low-grade gliomas, and a specificity of $61.7 \%$ and sensitivity of $58.3 \%$ for diagnosing high-grade gliomas. Similarly, in a survey of members of the American Society of Pediatric Neurosurgery (ASPN) and the AANS/CNS Joint Section for Pediatric Neurosurgery, Hankinson et al. ${ }^{24}$ found significant interrater heterogeneity with respect to labeling a brainstem lesion as typical or atypical for a DIPG, concluding that diagnosis of a DIPG based on radiological and clinical characteristics alone is often insufficient. Adjunctive imaging technologies, such as PET, have been proposed, but a recent study found that MRI and PET yielded discordant findings in $43 \%$ of patients and that even when the two modalities correlated with each other, there were sometimes unexpected histological findings. ${ }^{34}$ In this context, and 
TABLE 4. Final pathologic diagnoses ( $\mathrm{n}=58$ patients)

\begin{tabular}{cc}
\hline \multicolumn{1}{c}{ Pathology } & No. of Diagnoses $(\%)^{*}$ \\
\hline Glioma & \\
\hline Grade I & $11(18.6)$ \\
\hline Grade II & $10(16.9)$ \\
\hline Grade III-IV & $20(33.9)$ \\
\hline Ganglioglioma & $4(6.8)$ \\
\hline ETANTR/ETMR & $4(6.8)$ \\
\hline Therapy-related change & $2(3.4)$ \\
\hline Angiocentric glioma & $1(1.7)$ \\
\hline ATRT & $1(1.7)$ \\
\hline Germinoma & $1(1.7)$ \\
\hline PNET & $1(1.7)$ \\
\hline RGNT & $1(1.7)$ \\
\hline Edema & $1(1.7)$ \\
\hline Hamartoma & $1(1.7)$ \\
\hline Infarct & $1(1.7)$ \\
\hline
\end{tabular}

ETANTR = embryonal tumor with abundant neuropil and true rosettes; ETMR = embryonal tumor with multilayered rosettes; PNET = primitive neuroectodermal tumor; RGNT = rosette-forming glioneural tumor.

* One patient underwent 2 procedures with an intervening period of $>10$ years; ultimately, 2 diagnoses were obtained, for a total of 59 diagnoses in 58 patients.

particularly given the possibility of a nonneoplastic diagnosis, the standard practice of empirical radiation therapy has been questioned. ${ }^{29,35-37}$

Aside from the need for diagnostic certainty, there has been increased recognition that tissue samples will be needed for the development and testing of cutting-edge treatment options, including new chemotherapies, gene therapies, and immunotherapies. ${ }^{25,38}$ New treatments are especially necessary for patients with DIPG for whom meaningful therapeutic options are limited, and some have argued that progress has been impeded by the lack of available tissue for analysis. ${ }^{24}$ In particular, molecular profiling of biopsy specimens may accelerate the development and testing of targeted therapies and will likely guide future clinical trials., ${ }^{46,29,39-41}$ Technological advances have allowed molecular profiling to be performed on small biopsy specimens and have culminated in the realization that DIPGs actually represent distinct molecular subgroups that differ from other pediatric gliomas, providing insight into the failure of numerous treatment modalities to date. ${ }^{4,42-44}$

Despite the ongoing controversy over whether to biopsy a suspected DIPG or not, there is general consensus that a biopsy is warranted in patients with an atypical clinical history or whose MRI demonstrates a lesion with atypical radiological features, such as a focal appearance and/or abnormal contrast enhancement. ${ }^{25}$ "Liquid biopsy"-identifying molecular markers in the plasma and/or CSF-may be a viable alternative in the future, but this method does not yet supplant the histological and molecular data that can be obtained from a surgical biopsy. ${ }^{45}$ Therefore, our objective in this study was to provide an updated overview of the safety and efficacy of performing a brainstem
Procedure 1

Procedure 2
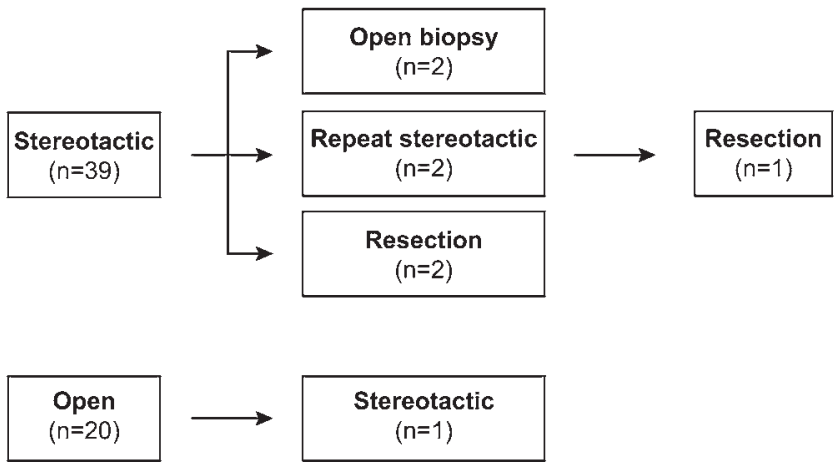

Endoscopic

$(n=1)$

FIG. 4. Flowchart demonstrating the number and types of additional procedures that followed the initial biopsy.

biopsy in children and young adults in this series of 58 patients undergoing 65 biopsies. Although brainstem biopsy has been described by prior authors, our goal was to redefine the morbidity and yield of this procedure in the modern era of frameless navigation, advanced preoperative imaging, and iMRI. In particular, we sought to share the workflow for our multidisciplinary approach to brainstem lesions in pediatric patients and young adults.

\section{Safety}

Safety remains a key concern among those who recommend against performing a brainstem biopsy. Potential complications include worsening of the patient's neurological status (including new or worsening eye movement abnormalities, hemiparesis, and/or facial paresis), hydrocephalus, pseudomeningocele/CSF leak, and wound infection. ${ }^{25,46,47}$ When weighing the risks and benefits of this procedure, high transient morbidity rates of $28 \%$, permanent morbidity rates of $9 \%$, and mortality rates of $4 \%$ cited by some studies have led to the conclusion that a brainstem biopsy is too dangerous. ${ }^{25,47-49}$ Other investigators, however, have confirmed that stereotactic biopsies are associated with relatively low rates of morbidity. $7,11,12$, ${ }^{33,50}$ A meta-analysis of 1480 brainstem biopsies identified an overall morbidity of $7.8 \%$, but a permanent morbidity of only $1.7 \%$ and a mortality of $0.9 \% .{ }^{29}$ The authors concluded that stereotactic brainstem biopsies should be considered just as safe and as accurate as biopsies of other regions of the brain. Similarly, a meta-analysis of 735 biopsies found an overall morbidity of $6.7 \%$, a permanent morbidity of $0.6 \%$, and a mortality of $0.6 \% .^{4}$ Among the 65 biopsies performed in the current series, there were 5 cases $(7.7 \%)$ of new or worsening neurological issues postoperatively. Only 2 of these resulted in persistent morbidity, with both cases involving stereotactic biopsies of a pontine lesion. The remaining 3 cases were all transient in nature.

In our opinion, both the biopsy target and trajectory need to be carefully planned with the assistance of an experienced neuroradiologist. The goal is to achieve an op- 
timal balance between diagnostic yield and safety. Neurovascular structures with a midline or deep locationsuch as the facial colliculus, cranial nerve nuclei, medial longitudinal fasciculus, corticospinal bundle, prominent perforating vessels, and crossing pial boundaries-should be avoided. It is important to assess the trajectory to the target in order to avoid the fibers of the facial nerve. The trajectory should ideally not cross the plane of the facial nerve root exit zone. Facial paralysis is particularly problematic because of the psychological impact brought about by disfigurement and the risk of corneal injury, speech impairment, and oral incontinence. Lastly, some low-grade gliomas (pilocytic astrocytomas and gangliogliomas) can have a firm consistency or be calcified, which can result in deflection of the tip of the biopsy needle unbeknownst to the surgeon, particularly with the use of an optical neuronavigational system, which tracks the top of the biopsy needle, and may therefore provide false reassurance that the needle is within the target, as opposed to an electromagnetic (EM)-based stereotactic system, which tracks the tip of the instrument. Currently, there is no EM-based biopsy needle.

\section{Efficacy}

Within our series, the overall diagnostic yield was 95.5\% (21 of 22) for open biopsies and 83.3\% (35 of 42) for stereotactic biopsies. A total of 8 additional procedures were required in order to obtain a final diagnosis following 1 open and 7 stereotactic cases (Fig. 4). Ultimately, a diagnosis was obtained in every case. A nonneoplastic diagnosis was confirmed in a total of 4 cases, and included deep white matter edema, an infarct, and 2 instances of treatment-related changes. Other authors have reported diagnostic yields of stereotactic biopsy that have ranged from $53 \%$ to $100 \%{ }^{7,11,12,17,25,33-35,46,47,51-53}$ A systematic review and meta-analysis of 1480 brainstem biopsies found an overall $96.2 \%$ rate of diagnostic success, while a second meta-analysis of 735 cases also found that adequate tissue sampling was obtained in $96.1 \%$ of cases. ${ }^{4,29}$ The lower diagnostic yield in the current series may reflect an inherent selection bias, given that our institution is a national and international referral center for neuro-oncology, and thus patients with challenging lesions comprise a significant portion of our patient population.

Of note, the majority of biopsy targets in the current series were chosen on the basis of contrast enhancement, DWI, or the ability to access an exophytic component of the tumor. However, in an effort to increase the diagnostic yield even further, we, along with a number of other groups, are now also using complementary imaging techniques in some cases, including perfusion-weighted imaging, proton MR spectroscopy, and PET, to identify biopsy sites based on chemical and metabolic features of the tumor. $29,34,35,54,55$

Additionally, nearly a third of the patients in this series were treated with molecularly targeted therapies, highlighting the expanding role of surgical biopsies. While biopsy tissue allows for a histopathological analysis, molecular analysis of the tissue via next-generation sequencing has the potential to yield not only molecular markers for diagnosis, but also increasingly relevant molecular targets for therapy.

\section{Study Limitations}

This study has several limitations related to its retrospective nature. Due to the lack of a standardized prospective protocol, the specifics of each biopsy-including the precise target, the number of samples that were obtained, and whether an iMRI was performed-were determined by the primary surgeon and the unique circumstances of each patient. Nevertheless, the fact that $92 \%$ of the procedures were performed by 1 of 2 surgeons limits the inherent variability in surgical technique and decision-making. Additionally, our study includes both open and stereotactic biopsies, which clearly are quite different procedures with respect to their safety profiles, as well as their likelihood of producing a diagnostic sample. Nevertheless, in our opinion it is clinically relevant to understand our approach to children and young adults with brainstem lesions, part of which involves deciding who would benefit up front from an open biopsy and who is a good candidate for a stereotactic biopsy. Therefore, where relevant, we distinguished between the 2 groups of procedures. Furthermore, due to the retrospective nature of this study, it is impossible, in hindsight, to identify the suspected diagnosis prior to each biopsy. This limits our ability to determine how frequently the ultimate diagnosis was different from the suspected diagnosis. Nevertheless, we maintain that a definitive tissue and molecular diagnosis allows for a more informed discussion regarding treatment and prognosis. A prospective study in which the suspected diagnosis is recorded prior to the biopsy is an interesting consideration for future work.

\section{Conclusions}

Brainstem lesions are challenging to diagnose and treat due to the critical neurovascular structures located in this region. However, with selection of the proper patient, target, and approach, brainstem biopsies can be accomplished with a high degree of safety and efficacy. A multidisciplinary approach to target selection is critical to a successful outcome. Additionally, iMRI has proven to be a valuable technology in these patients.

\section{Acknowledgments}

The Jackson Fogelman Pediatric Neurosurgery Research Fund supported this project. The authors wish to thank Andrew J. Gienapp (Neuroscience Institute, Le Bonheur Children's Hospital, and Department of Neurosurgery, University of Tennessee Health Science Center, Memphis, TN) for copy editing and publication assistance.

\section{References}

1. Freeman CR, Farmer JP. Pediatric brain stem gliomas: a review. Int J Radiat Oncol Biol Phys. 1998;40(2):265-271.

2. Laigle-Donadey F, Doz F, Delattre JY. Brainstem gliomas in children and adults. Curr Opin Oncol. 2008;20(6):662-667.

3. Recinos PF, Sciubba DM, Jallo GI. Brainstem tumors: where are we today? Pediatr Neurosurg. 2007;43(3):192-201.

4. Hamisch C, Kickingereder P, Fischer M, et al. Update on the diagnostic value and safety of stereotactic biopsy for pediatric brainstem tumors: a systematic review and meta-analysis of 735 cases. J Neurosurg Pediatr. 2017;20(3):261-268.

5. Rosenfeld A, Etzl M, Bandy D, et al. Use of positron emission tomography in the evaluation of diffuse intrinsic brain- 
stem gliomas in children. J Pediatr Hematol Oncol. 2011; 33(5):369-373.

6. Warren KE. Diffuse intrinsic pontine glioma: poised for progress. Front Oncol. 2012;2:205.

7. Abernathey CD, Camacho A, Kelly PJ. Stereotaxic suboccipital transcerebellar biopsy of pontine mass lesions. $\mathrm{J} \mathrm{Neu-}$ rosurg. 1989;70(2):195-200.

8. Epstein F. Intrinsic brain stem tumors of childhood. Surgical indications. Prog Exp Tumor Res. 1987;30:160-169.

9. Epstein F, McCleary EL. Intrinsic brain-stem tumors of childhood: surgical indications. J Neurosurg. 1986;64(1): 11-15.

10. Pérez-Gómez JL, Rodríguez-Alvarez CA, Marhx-Bracho A, Rueda-Franco F. Stereotactic biopsy for brainstem tumors in pediatric patients. Childs Nerv Syst. 2010;26(1):29-34.

11. Pincus DW, Richter EO, Yachnis AT, et al. Brainstem stereotactic biopsy sampling in children. J Neurosurg. 2006;104(2) (suppl):108-114.

12. Rajshekhar V, Chandy MJ. Computerized tomography-guided stereotactic surgery for brainstem masses: a risk-benefit analysis in 71 patients. J Neurosurg. 1995;82(6):976-981.

13. Rajshekhar V, Chandy MJ. Tuberculomas presenting as isolated intrinsic brain stem masses. Br J Neurosurg. 1997;11(2): 127-133.

14. Klimo P Jr, Nesvick CL, Broniscer A, et al. Malignant brainstem tumors in children, excluding diffuse intrinsic pontine gliomas. J Neurosurg Pediatr. 2016;17(1):57-65.

15. Klimo P Jr, Pai Panandiker AS, Thompson CJ, et al. Management and outcome of focal low-grade brainstem tumors in pediatric patients: the St. Jude experience. J Neurosurg Pediatr. 2013;11(3):274-281.

16. Coffey RJ, Lunsford LD. Diagnosis and treatment of brainstem mass lesions by CT-guided stereotactic surgery. Appl Neurophysiol. 1985;48(1-6):467-471.

17. Coffey RJ, Lunsford LD. Stereotactic surgery for mass lesions of the midbrain and pons. Neurosurgery. 1985;17(1): 12-18.

18. Franzini A, Allegranza A, Melcarne A, et al. Serial stereotactic biopsy of brain stem expanding lesions. Considerations on 45 consecutive cases. Acta Neurochir Suppl (Wien). 1988;42: 170-176.

19. Giunta F, Grasso G, Marini G, Zorzi F. Brain stem expanding lesions: stereotactic diagnosis and therapeutical approach. Acta Neurochir Suppl (Wien). 1989;46:86-89.

20. Gleason CA, Wise BL, Feinstein B. Stereotactic localization (with computerized tomographic scanning), biopsy, and radiofrequency treatment of deep brain lesions. Neurosurgery. 1978;2(3):217-222.

21. Kratimenos GP, Nouby RM, Bradford R, et al. Image directed stereotactic surgery for brain stem lesions. Acta Neurochir (Wien). 1992;116(2-4):164-170.

22. Albright AL, Packer RJ, Zimmerman R, et al. Magnetic resonance scans should replace biopsies for the diagnosis of diffuse brain stem gliomas: a report from the Children's Cancer Group. Neurosurgery. 1993;33(6):1026-1030.

23. Schumacher M, Schulte-Mönting J, Stoeter P, et al. Magnetic resonance imaging compared with biopsy in the diagnosis of brainstem diseases of childhood: a multicenter review. $\mathrm{J} \mathrm{Neu}$ rosurg. 2007;106(2)(suppl):111-119.

24. Hankinson TC, Campagna EJ, Foreman NK, Handler MH. Interpretation of magnetic resonance images in diffuse intrinsic pontine glioma: a survey of pediatric neurosurgeons. $J$ Neurosurg Pediatr. 2011;8(1):97-102.

25. Dellaretti M, Touzet G, Reyns N, et al. Correlation among magnetic resonance imaging findings, prognostic factors for survival, and histological diagnosis of intrinsic brainstem lesions in children. J Neurosurg Pediatr. 2011;8(6): 539-543.

26. Khuong-Quang DA, Buczkowicz P, Rakopoulos P, et al.
K27M mutation in histone H3.3 defines clinically and biologically distinct subgroups of pediatric diffuse intrinsic pontine gliomas. Acta Neuropathol. 2012;124(3):439-447.

27. Rutka JT. Biopsy of diffuse intrinsic pontine gliomas? J Neurosurg Pediatr. 2012;10(2):79-80.

28. MacDonald TJ. Diffuse intrinsic pontine glioma (DIPG): time to biopsy again? Pediatr Blood Cancer. 2012;58(4): 487-488.

29. Kickingereder P, Willeit P, Simon T, Ruge MI. Diagnostic value and safety of stereotactic biopsy for brainstem tumors: a systematic review and meta-analysis of 1480 cases. Neurosurgery. 2013;72(6):873-882.

30. Epstein F. A staging system for brain stem gliomas. Cancer. 1985;56(7)(suppl):1804-1806.

31. Stroink AR, Hoffman HJ, Hendrick EB, Humphreys RP. Diagnosis and management of pediatric brain-stem gliomas. $J$ Neurosurg. 1986;65(6):745-750.

32. Kornreich L, Schwarz M, Karmazyn B, et al. Role of MRI in the management of children with diffuse pontine tumors: a study of 15 patients and review of the literature. Pediatr Radiol. 2005;35(9):872-879.

33. Rachinger W, Grau S, Holtmannspötter M, et al. Serial stereotactic biopsy of brainstem lesions in adults improves diagnostic accuracy compared with MRI only. J Neurol Neurosurg Psychiatry. 2009;80(10):1134-1139.

34. Massager N, David P, Goldman S, et al. Combined magnetic resonance imaging- and positron emission tomography-guided stereotactic biopsy in brainstem mass lesions: diagnostic yield in a series of 30 patients. J Neurosurg. 2000;93(6): 951-957.

35. Pirotte BJ, Lubansu A, Massager N, et al. Results of positron emission tomography guidance and reassessment of the utility of and indications for stereotactic biopsy in children with infiltrative brainstem tumors. J Neurosurg. 2007;107(5) (suppl):392-399.

36. Krieger MD, Blüml S, McComb JG. Magnetic resonance spectroscopy of atypical diffuse pontine masses. Neurosurg Focus. 2003;15(1):E5.

37. St George EJ, Walsh AR, Sgouros S. Stereotactic biopsy of brain tumours in the paediatric population. Childs Nerv Syst. 2004;20(3):163-167.

38. Jeuken JW, van der Maazen RW, Wesseling P. Molecular diagnostics as a tool to personalize treatment in adult glioma patients. Technol Cancer Res Treat. 2006;5(3):215-229.

39. Paugh BS, Broniscer A, Qu C, et al. Genome-wide analyses identify recurrent amplifications of receptor tyrosine kinases and cell-cycle regulatory genes in diffuse intrinsic pontine glioma. J Clin Oncol. 2011;29(30):3999-4006.

40. Bartels U, Hawkins C, Vézina G, et al. Proceedings of the diffuse intrinsic pontine glioma (DIPG) Toronto Think Tank: advancing basic and translational research and cooperation in DIPG. J Neurooncol. 2011;105(1):119-125.

41. Zarghooni M, Bartels U, Lee E, et al. Whole-genome profiling of pediatric diffuse intrinsic pontine gliomas highlights platelet-derived growth factor receptor alpha and poly (ADPribose) polymerase as potential therapeutic targets. J Clin Oncol. 2010;28(8):1337-1344.

42. Buczkowicz P, Hoeman C, Rakopoulos P, et al. Genomic analysis of diffuse intrinsic pontine gliomas identifies three molecular subgroups and recurrent activating ACVR1 mutations. Nat Genet. 2014;46(5):451-456.

43. Grasso CS, Tang Y, Truffaux N, et al. Functionally defined therapeutic targets in diffuse intrinsic pontine glioma. Nat Med. 2015;21(7):827.

44. Schroeder KM, Hoeman CM, Becher OJ. Children are not just little adults: recent advances in understanding of diffuse intrinsic pontine glioma biology. Pediatr Res. 2014;75(1-2): 205-209.

45. Lu VM, Power EA, Zhang L, Daniels DJ. Liquid biopsy for 
diffuse intrinsic pontine glioma: an update. J Neurosurg Pediatr. 2019;24(5):593-600.

46. Roujeau T, Machado G, Garnett MR, et al. Stereotactic biopsy of diffuse pontine lesions in children. J Neurosurg. 2007; 107(1)(suppl):1-4.

47. Steck J, Friedman WA. Stereotactic biopsy of brainstem mass lesions. Surg Neurol. 1995;43(6):563-568.

48. Cartmill M, Punt J. Diffuse brain stem glioma. A review of stereotactic biopsies. Childs Nerv Syst. 1999;15(5):235-238.

49. Guillamo JS, Doz F, Delattre JY. Brain stem gliomas. Curr Opin Neurol. 2001;14(6):711-715.

50. Kreth FW, Muacevic A, Medele R, et al. The risk of haemorrhage after image guided stereotactic biopsy of intra-axial brain tumours - a prospective study. Acta Neurochir (Wien). 2001;143(6):539-546.

51. Aker FV, Hakan T, Karadereler S, Erkan M. Accuracy and diagnostic yield of stereotactic biopsy in the diagnosis of brain masses: comparison of results of biopsy and resected surgical specimens. Neuropathology. 2005;25(3):207-213.

52. Kondziolka D, Lunsford LD. Results and expectations with image-integrated brainstem stereotactic biopsy. Surg Neurol. 1995;43(6):558-562.

53. Kesari S, Kim RS, Markos V, et al. Prognostic factors in adult brainstem gliomas: a multicenter, retrospective analysis of 101 cases. J Neurooncol. 2008;88(2):175-183.

54. Leach PA, Estlin EJ, Coope DJ, et al. Diffuse brainstem gliomas in children: should we or shouldn't we biopsy? $\mathrm{Br} J \mathrm{Neu}$ rosurg. 2008;22(5):619-624.

55. Pirotte B, Goldman S, Massager N, et al. Comparison of ${ }^{18} \mathrm{~F}$ -
FDG and ${ }^{11} \mathrm{C}$-methionine for PET-guided stereotactic brain biopsy of gliomas. J Nucl Med. 2004;45(8):1293-1298.

\section{Disclosures}

The authors report no conflict of interest concerning the materials or methods used in this study or the findings specified in this paper.

\section{Author Contributions}

Conception and design: Hersh, Klimo. Acquisition of data: Hersh, Kumar, Moore, Vaughn. Analysis and interpretation of data: all authors. Drafting the article: Hersh, Kumar. Critically revising the article: all authors. Reviewed submitted version of manuscript: all authors. Approved the final version of the manuscript on behalf of all authors: Hersh. Administrative/technical/material support: Vaughn, Klimo. Study supervision: Klimo.

\section{Supplemental Information}

Previous Presentations

This work was presented as an oral presentation at the AANS/ CNS Section on Pediatric Neurological Surgery at its 2019 Annual Meeting in Scottsdale, Arizona.

\section{Correspondence}

David S. Hersh: Connecticut Children's, Hartford, CT. dhersh@connecticutchildrens.org. 